\title{
Infarto cerebral limítrofe secundario a enfermedad inflamatoria orbitaria idiopática
}

Watershed cerebral infarction secondary to idiopathic orbital inflammatory disease

\author{
Jorge A. Ramírez-Quiñones ${ }^{1, a}$, Danny M. Barrientos-Imán ${ }^{1, a}$, Diana Rivas-Franchini ${ }^{2, ~ b}$, Ana M. \\ Valencia-Chávez ${ }^{1, a}$, Carlos Abanto-Argomedo ${ }^{1, a}$ \\ ${ }^{1}$ Departamento de Investigación, Docencia y Atención Especializada en Enfermedades Neurovasculares y Metabólicas. Instituto Nacional de Ciencias Neurológicas. Lima, Perú. \\ ${ }^{2}$ Departamento de Neuropatología. Instituto Nacional de Ciencias Neurológicas. Lima, Perú. \\ ${ }^{a}$ Médico Neurólogo. \\ ${ }^{\mathrm{b}}$ Médico Patólogo.
}

An Fac med. 2018;79(1):44-48 / http://dx.doi.org/10.15381/anales.v79i1.14591

\section{Correspondencia:}

Jorge Alonso Ramirez Quiñones

alonsor45@hotmail.com

Instituto Nacional de Ciencias

Neurológicas. Lima, Perú.

Dirección: Jr. Ancash 1271 Lima 1 Perú.

Teléfono: 4117700 - Anexo 232

Recibido: 16 enero 2018

Aceptado: 6 abril 2018

Conflictos de interés: Los autores declaran no tener conflictos de interés en la publicación del presente artículo.

El resumen de este caso fue presentado en póster y presentado de manera oral en el $18^{\circ}$ congreso de la Sociedad Iberoamericana de Enfermedad Cerebrovascular (SIECV) realizado el año 2015 en Lima, Perú.

Fuentes de financiamiento:

Autofinanciado

Citar como: Ramírez-Quiñones J, Barrientos-Imán D, Rivas-Franchini D, Valencia-Chávez A, Abanto-Argomedo C. Infarto cerebral limítrofe secundario a enfermedad inflamatoria orbitaria idiopática. An Fac med. 2018;79(1):44-48 DOl: http://dx.doi.org/10.15381/anales. v79i1.14591

\section{Resumen}

Los infartos limitrofes son aquellos ubicados en regiones entre dos territorios vasculares contiguos y la enfermedad inflamatoria orbitaria idiopática es un proceso inflamatorio orbitario inespecifico. Presentamos el caso de un varón de 60 años con exoftalmos, ptosis palpebral, disminución de agudeza visual derecha y afección de los nervios oculomotores derechos. Los estudios de imágenes mostraron una masa retro ocular derecha con invasión de los senos cavernosos y esfenoidal. Durante la hospitalización, el paciente sufrió un infarto cerebral limitrofe por compresión de la arteria carótida interna derecha en su segmento intracavernoso. La exéresis y la anatomía patológica de la lesión orbitaria fueron compatibles con la enfermedad inflamatoria orbitaria idiopática. Nuestro caso muestra a la enfermedad inflamatoria orbitaria idiopática como una causa inhabitual de infarto cerebral limitrofe.

Palabras clave: Infarto Cerebral; Seudotumor Orbitario; Seno Cavernoso; Trastornos Cerebrovasculares (fuente: DeCS BIREME).

\section{Abstract}

Watershed cerebral infarctions are those located in regions between two adjacent vascular territories and Idiopathic orbital inflammatory disease is a nonspecific inflammatory process of the orbit. We report the case of a 60-year-old man who presented suddenly in the right eye: exophthalmos, ptosis, reduced visual acuity and paresis of the extraocular muscles innervated by the III, IV and VI cranial nerves. Imaging studies showed a right retro-ocular mass with invasion of the ipsilateral cavernous and sphenoid sinus. During hospitalization, suddenly he presented a watershed infarction with narrowing of the right internal carotid artery in its intracavernous portion. Surgical excision of the lesion was performed and pathology showed a characteristic infiltration of idiopathic orbital inflammatory disease. Our case shows idiopathic orbital inflammatory disease as an uncommon cause of watershed cerebral infarction.

Keywords: Cerebral Infarction; Orbital Pseudotumor; Cavernous Sinus; Cerebrovascular Disorders (source MeSH NLM). 


\section{INTRODUCCION}

Los infartos cerebrales limítrofes (IL) representan alrededor del $12 \%$ de todos los infartos y se ubican en regiones entre dos territorios vasculares contiguos ${ }^{(1,2)}$. Son clasificados según su localización en internos y externos, siendo los primeros causados principalmente por un mecanismo hemodinámico relacionado a una estenosis de la arteria carótida interna $(\mathrm{ACl})$ ipsilateral ${ }^{(3-6)}$. La enfermedad inflamatoria orbitaria idiopática (EIOI) representa una causa inhabitual de infarto cerebral limítrofe de mecanismo hemodinámico secundario a la compresión de la $\mathrm{ACl}$ en el seno cavernoso. Reportamos el caso de un paciente peruano con un infarto cerebral limítrofe interno producido por una EIOI confirmada por el estudio histopatológico.

\section{CASO CLÍNICO}

Paciente varón de 60 años de edad, natural y procedente de Lima, sin antecedentes familiares de enfermedad neurológica y con diagnóstico previo de hipertensión arterial y diabetes mellitus tipo 2 con control adecuado. Refirió que 3 días antes presentó súbitamente dolor orbitario derecho de intensidad moderada asociado a enrojecimiento conjuntival e hinchazón de la región ocular ipsilateral, un día después se añadió disminución de agudeza visual en ojo derecho y el día del ingreso se añadió diplopía, caída del párpado superior derecho y dificultad para mover el ojo derecho. Al examen físico general no se encontraron hallazgos contributorios. Al examen regional se evidenció exoftalmos y edema palpebral en ojo derecho asociado a inyección conjuntival. Al examen neurológico: despierto, orientado en tiempo, espacio y persona, sin compromiso motor ni sensitivo, tono muscular y reflejos osteotendinosos conservados, agudeza visual en ojo derecho: cuenta dedos a 1 metro y en ojo izquierdo: normal, paresia de los músculos extraoculares inervados por el III, IV y VI nervios craneales derechos, hipoestesia en región correspondiente a V1 derecho, resto sin alteraciones. Los exámenes de apoyo al diagnóstico: hematocrito, hemoglobina, fórmula leu- cocitaria, recuento plaquetario, perfil de coagulación, glucosa, función hepática y renal, electrolitos y perfil tiroideo fueron normales. Las pruebas serológicas para sífilis y HIV fueron negativas. Radiografía de tórax sin anormalidades. La tomografía espiral multicorte (TEM) de órbitas sin contraste mostró una masa isodensa en la región retro ocular derecha que compromete a los músculos extraoculares y al nervio óptico ipsilateral con invasión del seno cavernoso y del seno esfenoidal ipsilateral (Figura 1). La resonancia magnética (RM) de órbitas evidenció lesión de similares características con captación de contraste de forma heterogénea.

Durante la hospitalización, de forma súbita presentó debilidad muscular y disminución de la sensibilidad superficial ambos en hemicuerpo izquierdo, además de dificultad leve para articular las palabras. Se realizó una RM cerebral con difusión que mostró áreas isquémicas correspondientes con un IL interno de tipo parcial entre los territorios correspondientes a la arteria cerebral anterior (ACA) y la arteria cerebral media (ACM) del lado derecho (Figura 2A). La angiotomografía evidenció una marcada disminución del calibre de la $\mathrm{ACl}$ derecha en su segmento intracavernoso (Figura 2B y 2C). Una nueva RM de órbitas mostró el efecto compresivo de la masa sobre la $\mathrm{ACl}$ derecha, disminuyendo el lumen de forma marcada comparado con la $\mathrm{ACl}$ izquierda. La ecografía carotidea, el ecocardiograma transtorácico y el Holter de
24 horas fueron negativos. Se practicó la exéresis quirúrgica de la lesión con fines diagnósticos y terapéuticos. La anatomía patológica mostró un infiltrado inespecífico característico de la EIOI (Figura 3). Luego de superar el periodo agudo del infarto cerebral, se inició tratamiento con prednisona $1 \mathrm{mg} / \mathrm{kg}$. Durante el seguimiento tuvo una evolución clínica y radiológica favorable, sin recurrencia de enfermedad cerebrovascular y disminución del tamaño de la lesión por RM de órbitas.

\section{DISCUSIÓN}

Los infartos limítrofes (IL) cerebrales se definen como lesiones isquémicas ubicadas en una región entre dos territorios vasculares contiguos. Son clásicamente clasificados según su localización como infartos externos (corticales) e internos (subcorticales o profundos). Los IL externos se localizan en las regiones ubicadas entre los territorios de la ACA y la ACM (anterior y paramediano) y entre los territorios de la ACM y la ACP (posterior). Los IL internos se localizan en las regiones ubicadas entre las ramas corticales de la ACA, ACM o ACP y las ramas profundas como las arterias lenticuloestriadas (mediales y laterales) y la arteria coroidea anterior (1). Según un estudio realizado por Yong et al utilizando la resonancia magnética por difusión, el 12,7\% de los infartos evaluados estuvieron en territorios limítrofes ${ }^{(2)}$. Los IL más comunes son aquellos ubicados en-

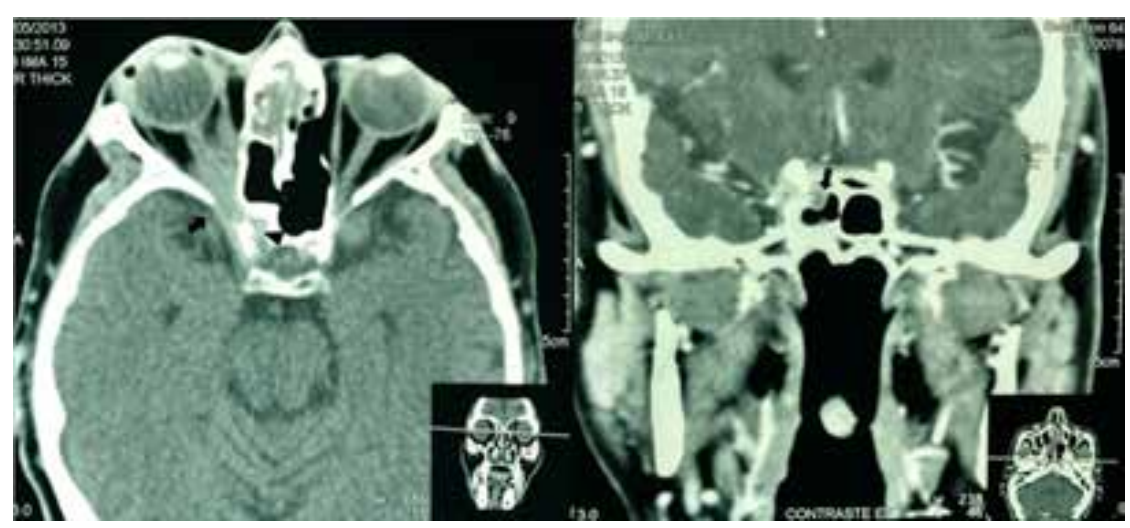

Figura 1. TEM de órbitas. Izquierda: Corte axial que muestra masa retro orbitaria derecha con extensión hacia el seno cavernoso a través de la fisura orbitaria superior (flecha) y extensión a través del canal óptico (cabeza de flecha). Derecha: Corte coronal que muestra extensión hacia el seno esfenoidal (flecha). 


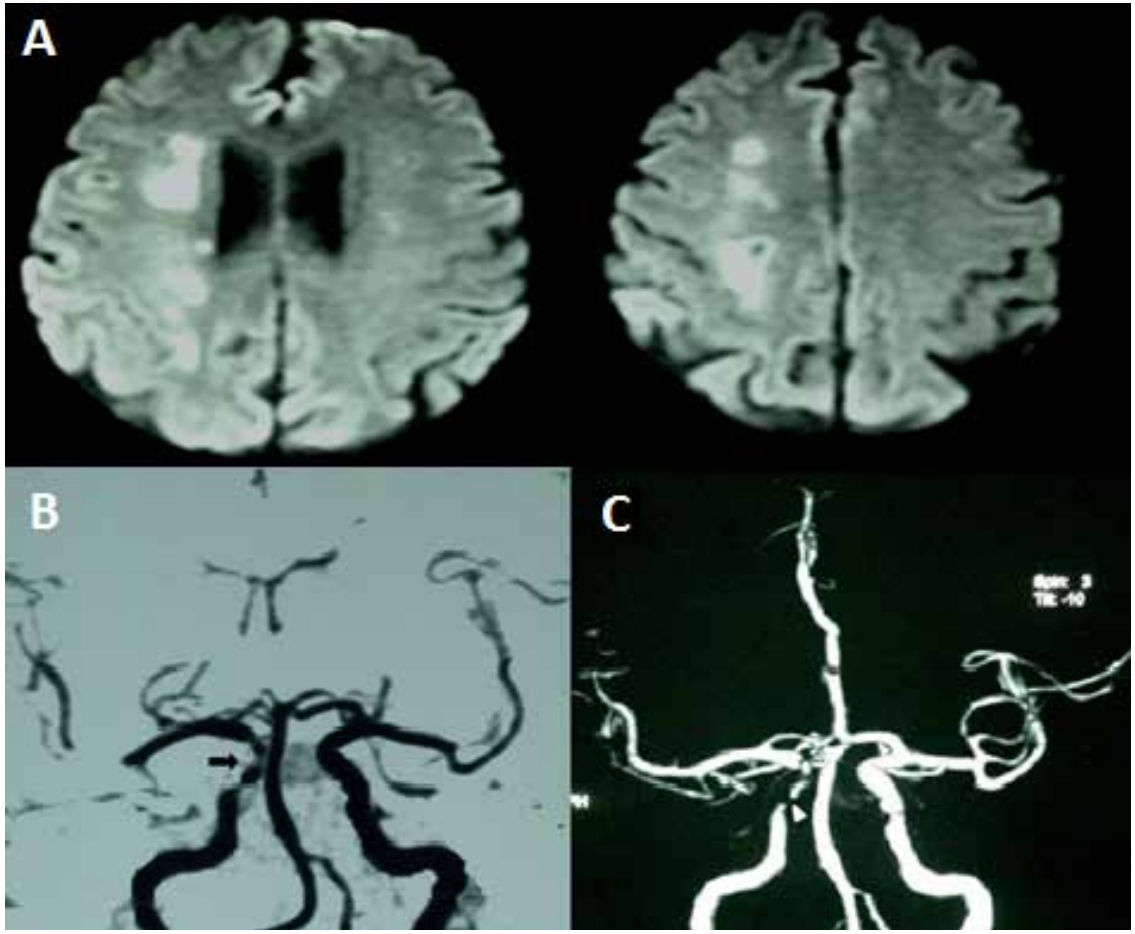

Figura 2. RM cerebral protocolo difusión y Angiotomografía Cerebral. A) RM cerebral protocolo difusión que muestra infarto cerebral en territorio limítrofe interno con patrón "rosario" entre los territorios de ACA y ACM del lado derecho. B) Angio-tomografía de vasos intracraneales muestra marcada estenosis de la ACl derecha a nivel del seno cavernoso (flecha). C) Reconstrucción 3D de angiotomografía con hallazgo ya descrito (cabeza de flecha).

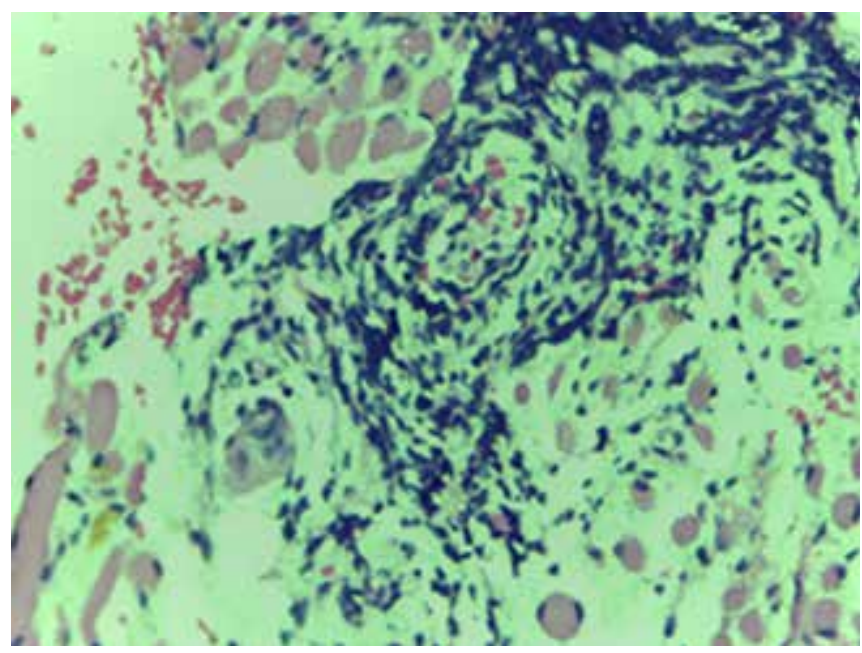

Figura 3. Histología. Se observa fibras musculares pequeñas separadas por infiltrado inflamatorio crónico a predominio linfoplasmacitario y escasos fibroblastos. (Hematoxilina-eosina x200).

tre el territorio irrigado por los segmentos terminales de las arterias lenticuloestriadas y las ramas medulares penetrantes piales de la $\mathrm{ACM}^{(3)}$.

Anatómicamente, los IL comprometen las zonas distales de dos territorios arteriales no anastomóticos ${ }^{(4)}$. Existen dos frecuencia, de una de las arterias intracraneales mayores (ACM, ACA, ACM) con un episodio de hipotensión sistémica, dicha zona con mayor susceptibilidad sufrirá un daño isquémico ${ }^{(5,6)}$. La segunda es la teoría de la microembolia, que se basa en la presencia de microémbolos de origen cardíaco o arterial que obstruyen el flujo sanguíneo normal en las arterias afectadas, mecanismo demostrado por el estudio de Pollanen y Deck ${ }^{(7)}$. Algunos autores sugieren que de forma frecuente los IL son el resultado de la interacción de ambos mecanismos ${ }^{(8)}$.

Aunque definir un mecanismo fisiopatológico específico para los IL según su localización es difícil, se considera que existe una mayor asociación de los IL internos con el mecanismo hemodinámico $y$, con una asociación menos contundente, de los IL externos con el mecanismo microembólico; aunque puede haber excepciones o mecanismos combinados en cada caso ${ }^{(3,4)}$. En nuestro caso la localización del infarto y la disminución del calibre de la $\mathrm{ACl}$ ipsilateral secundario a un fenómeno compresivo apoyan un mecanismo hemodinámico.

El compromiso de la $\mathrm{ACl}$ es casi patognomónico en los IL internos y en la mayoría de los casos su obstrucción es interna (intraluminal) teniendo como etiologías más comunes a las placas ateroescleróticas y la disección arterial. El compromiso externo (compresivo) es raro, siendo pocas las causas reportadas que han causado un IL destacando por frecuencia las neoplasias de cabeza y cuello (meningioma, adenoma de hipófisis, histiocitoma fibrosos y neurilemoma del $X$ nervio craneal) ${ }^{(9,10)}$, la trombosis del seno cavernoso ${ }^{(11)}$ y la mucormicosis rino-orbito-cerebral ${ }^{(12)}$; casi todas ellas comprometiendo a la $\mathrm{ACl}$ en su segmento intracavernoso. Existen escasos reportes de la EIOI como causa de enfermedad cerebrovascular (accidente isquémico transitorio o infarto cerebral) por compresión de la $\mathrm{ACl}$ $(13,14,15)$. Se realizó una búsqueda bibliográfica en Medline usando los términos "orbital pseudotumor", "idiopathic orbital inflammatory disease", "idiopathic orbital inflammation", "cerebral infarction", "ischemic stroke", "watershed infarction" y "borderzone infarct"; y en Lilacs y Scielo usando los términos "seudotumor orbi- 
tario", "enfermedad inflamatoria orbitaria idiopática", "infarto cerebral" y "enfermedad cerebrovascular isquémica" no obteniendo resultados en nuestra región que asocie ambas patologías, siendo este el primer reporte latinoamericano.

La EIOI, también conocido como pseudotumor orbitario, es un proceso inflamatorio inespecífico de la órbita ${ }^{(16)}$, que según los datos obtenidos por Shikishima et al representa hasta el 18\% de los "tumores" en dicha región ${ }^{(17)}$. Es una enfermedad de etiología desconocida, aunque se considera que en su patogénesis interaccionan de forma compleja factores genéticos, inmunes $y$, en algunos casos, infecciosos ${ }^{(18)}$. Histológicamente se caracteriza por un infiltrado inflamatorio constituido por polimorfonucleares, linfocitos, células plasmáticas, macrófagos y eosinófilos con aumento del tejido conectivo circundante, edema y fibrosis ${ }^{(18,19)}$. En la mayoría de casos, la lesión está circunscrita a la órbita y puede comprometer cualquiera de las estructuras anatómicas presentes produciendo miositis, dacrioadenitis, perineuritis, periescleritis, perivasculitis o un proceso difuso que compromete varias estructuras (20). De forma infrecuente, como ocurrió en nuestro caso, la EIOI se extiende a través de la fisura orbitaria superior, la fisura orbitaria inferior o el canal óptico hacia otras regiones, siendo las preferidas el seno cavernoso y la fosa craneal media ${ }^{(20,21,22)}$.

Los dolores, la proptosis, el edema palpebral, la congestión conjuntival, la restricción de la motilidad ocular y la disminución de la agudeza visual encontrados en nuestro caso corresponden con la afectación de distintas estructuras dentro de la órbita y son hallazgos reportados de forma común en la EIOI ${ }^{(16,22)}$. De forma característica, la EIOI es de evolución subaguda, aunque han sido descritas formas de presentación rápidamente progresiva (como una emergencia oftalmológica) y formas crónicas ${ }^{(18)}$. Los hallazgos neurológicos como el compromiso de la fuerza y la sensibilidad en el hemicuerpo izquierdo, así como la disartria se produjeron por un daño del centro semioval derecho que incluye a axones motores eferentes $y$ axones sensitivos aferentes.
Para el diagnóstico topográfico de los IL se usa la RM por difusión, protocolo con capacidad de detectar con precisión la localización de la lesión isquémica minutos después del evento clínico. En los IL internos se han descrito dos subtipos radiológicos: 1) El confluente en el que la lesión es única con forma de "cigarro" y 2) el parcial o "con forma de rosario" caracterizado por lesiones múltiples continuas que tiene una asociación con el compromiso de la $\mathrm{ACl}$ ipsilateral como en nuestro caso ${ }^{(23)}$.

Para el diagnóstico de la EIOI las imágenes (que incluyen la TEM y la RM) son fundamentales ya que brindan información acerca de la localización, las estructuras afectadas y la extensión hacia estructuras extraorbitarias. En la tomografía, la EIOI se presenta como una masa focal o difusa, ligeramente hiperdensa, de bordes irregulares con captación de contraste no uniforme. En nuestro caso, además se encontró engrosamiento uveoescleral, ensanchamiento de los músculos extraoculares y del nervio óptico, captación de contraste en el espacio epiescleral y proptosis, hallazgos reportados en la literatura ${ }^{(18)}$. La RM detecta con mayor precisión la extensión extracraneal de las lesiones y la aplicación de contraste y el uso de los protocolos de saturación grasa y de difusión ayudan a diferenciarlas de otras lesiones. Además, en nuestro caso se utilizó la angiotomografía de vasos supra-aórticos e intracraneales para valorar la integridad del sistema arterial, con el hallazgo de una estenosis de la $\mathrm{ACl}$ a nivel del seno cavernoso producido por la EIOI. El diagnóstico definitivo de la EIOI es histológico caracterizado por un infiltrado inflamatorio no específico con diversas variantes como la esclerosante, la granulomatosa, la vasculítica y la eosinofílica ${ }^{(24)}$.

Como ocurrió en nuestro caso y de forma característica la mayoría de pacientes con EIOI tienen una buena respuesta al tratamiento con corticoides orales e intravenosos a altas dosis, con mejoría clínica a partir de las primeras 24 a 48 horas de tratamiento. La tasa de curación con corticoides oscila entre $30-40 \%$ y la de recurrencia entre $15-58 \%$ dependiendo del tipo histológico, las estructuras comprometidas y las condiciones médicas asociadas. En aquellos casos sin respuesta a corticoides se han utilizado la radioterapia, agentes inmunosupresores (azatioprina, metrotexate, micofenolato de mofetilo y ciclosporina) inmunoglobulina y anticuerpos monoclonales con resultados favorables ${ }^{(25)}$. La exéresis de la lesión ha sido utilizada en los casos con rápida invasión extraorbitaria, en aquellos sin diagnóstico definido como en nuestro caso y en aquellos con pobre respuesta al tratamiento médico (24). No existen guías uniformizadas de tratamiento, por lo que debe ser individualizado para cada paciente.

Nuestro caso muestra a la EIOI como una causa inhabitual de infarto cerebral limítrofe. Ante toda lesión ocupante de espacio en la órbita se recomienda un diagnóstico rápido y preciso con la finalidad de prevenir su extensión hacia otras regiones como el seno cavernoso y, de esta forma, disminuir el riesgo de infarto cerebral limítrofe de mecanismo hemodinámico por afectación compresiva de la arteria carótida interna.

\section{REFERENCIAS BIBLIOGRÁFICAS}

1. Del Sette M, Eliasziw M, Streifler JY, Hachinski VC, Fox AJ, Barnett HJ. Internal borderzone infarction: a marker for severe stenosis in patients with symptomatic internal carotid artery disease. For the North American Symptomatic Carotid Endarterectomy (NASCET) Group. Stroke. 2000;31(3):631-6. doi. org/10.1161/01.STR.31.3.631

2. Yong SW, Bang OY, Lee PH, Li WY. Internal and cortical border-zone infarction: clinical and diffusion-weighted imaging features. Stroke. 2006;37(3):841-6. doi.org/10.1161/01. STR.0000202590.75972.39

3. Mangla R, Kolar B, Almast J, Ekholm SE. Border zone infarcts: pathophysiologic and imaging characteristics. Radiographics. 2011;31(5):1201-14. doi.org/10.1148/rg.315105014

4. Momjian-Mayor I, Baron J-C. The pathophysiology of watershed infarction in internal carotid artery disease: review of cerebral perfusion studies. Stroke. 2005;36(3):567-77. doi.org/10.1161/01. STR.0000155727.82242.e1

5. Howard R, Trend P, Russell RW. Clinical features of ischemia in cerebral arterial border zones after periods of reduced cerebral blood flow. Arch Neurol. 1987;44(9):934-40. doi:10.1001/archneur.1987.00520210036016

6. Bladin CF, Chambers BR. Frequency and pathogenesis of hemodynamic stroke. Stroke. 1994;25(11):2179-82. doi.org/10.1161/01. STR.25.11.2179

7. Pollanen MS, Deck JH. The mechanism of embolic watershed infarction: experimental studies. Can J Neurol Sci. 1990;17(4):395-8. doi.org/10.1017/ S031716710003095X 
8. Caplan LR, Hennerici M. Impaired clearance of emboli (washout) is an important link between hypoperfusion, embolism, and ischemic stroke. Arch Neurol. 1998;55(11):1475-82. doi:10.1001/ archneur.55.11.1475

9. Komotar R, Keswani S, Wityk R. Meningioma presenting as stroke: report of two cases and estimation of incidence. J Neurol Neurosurg Psychiatry. 2003;74(1):136-7. doi.org/10.1136/jnnp.74.1.136

10. Matsuo R, Kamouchi M, Inoue T, Okada Y, Ibayashi S. Cerebral Infarction Due to Carotid Occlusion Caused by Cervical Vagal Neurilemmoma Report of a Case. Stroke. 2002;33(5):1428-31. doi. org/10.1161/01.STR.0000015241.40071.99

11. Suntrup S, Kemmling A, Dziewas R, Niederstadt T, Ritter MA. Septic cavernous sinus thrombosis complicated by occlusion of the internal carotid artery and multiple embolic strokes after surgery of an anorectal abscess: a clinical chameleon. Neurologist. 2012;18(5):310-2. doi: 10.1097/ NRL.0b013e31826752a6

12. Thajeb P, Thajeb T, Dai D. Fatal strokes in patients with rhino-orbito-cerebral mucormycosis and associated vasculopathy. Scand J Infect Dis. 2004;36(9):643-8. doi. org/10.1080/00365540410020794

13. Borruat FX, Vuilleumier P, Ducrey N, Fankhauser $H$, Janzer RC, Regli F. Idiopathic orbital inflam- mation (orbital inflammatory pseudotumour): an unusual cause of transient ischaemic attack. J Neurol Neurosurg Psychiatry. 1995;58(1):88-90. doi.org/10.1136/jnnp.58.1.88

14. Vuysere SD, Hermans R, Sciot R, Crevits I, Marchal G. Extraorbital Inflammatory Pseudotumor of the Head and Neck: CT and MR Findings in Three Patients. AJNR Am J Neuroradiol. 1999;20(6):1133-9.

15. Bhagwat B, Deshpande S, Ekbote S, Nalgirkar A, Sharma R. Case report: Sclerosing orbital inflammatory syndrome with intracranial extension. Indian J Radiol Imaging. 2000;10(3):161.

16. Yuen SJA, Rubin PAD. Idiopathic orbital inflammation: distribution, clinical features, and treatment outcome. Arch Ophthalmol. 2003;121(4):491-9. doi:10.1001/archopht.121.4.491

17. Shikishima K, Kawai K, Kitahara K. Pathological evaluation of orbital tumours in Japan: analysis of a large case series and 1379 cases reported in the Japanese literature. Clin Experiment Ophthalmol. 2006;34(3):239-44. DOI: 10.1111/j.14429071.2006.01192.x

18. Chaudhry IA, Shamsi FA, Arat YO, Riley FC. Orbital pseudotumor: distinct diagnostic features and management. Middle East Afr J Ophthalmol. 2008;15(1):17-27. DOI: 10.4103/0974-9233.53370

19. Gordon LK. Diagnostic dilemmas in orbital inflammatory disease. Ocul Immunol Inflamm.
2003;11(1):3-15. doi.org/10.1076/ocii.11.1.3.15577

20. Clifton AG, Borgstein RL, Moseley IF, Kendal $B E$, Shaw PJ. Intracranial extension of orbital pseudotumour. Clin Radiol. 1992;45(1):23-6. doi. org/10.1016/S0009-9260(05)81462-X

21. Lee EJ, Jung SL, Kim BS, Ahn KJ, Kim YJ, Jung AK et al. MR imaging of orbital inflammatory pseudotumors with extraorbital extension. Korean J Radiol. 2005;6(2):82-8. doi.org/10.3348/kjr.2005.6.2.82

22. Li Y, Lip G, Chong V, Yuan J, Ding Z. Idiopathic orbital inflammation syndrome with retro-orbita involvement: a retrospective study of eight patients. PloS One. 2013;8(2):e57126. doi.org/10.1371/ journal.pone.0057126

23. Li H-F, Zhang $X$, Zhang $Y$, Pan X-D, Zhao H-Q, $\mathrm{Li} \mathrm{H}$. Clinical and neuroradiological features of internal watershed infarction and the occlusive diseases of carotid artery system. Neurol Res. 2010;32(10):1090-6. doi.org/10.1179/01616411 0X12681290831324

24. Mombaerts I, Goldschmeding R, Schlingemann $\mathrm{RO}$, Koornneef L. What is orbital pseudotumor? Surv Ophthalmol. 1996;41(1):66-78. DOI: 10.1016/ S0039-6257(97)81996-0

25. Espinoza GM. Orbital inflammatory pseudotumors: etiology, differential diagnosis, and management. Curr Rheumatol Rep. 2010;12(6):443-7. doi org/10.1007/s11926-010-0128-8 